\title{
Parietal fibrinous peritonitis in cattle: A literature review
}

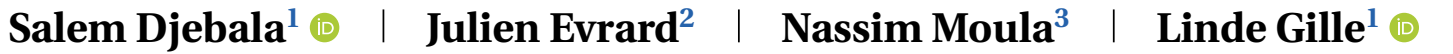 \\ Arnaud Sartelet $^{1}$ | Philippe Bossaert ${ }^{1}$
}

\author{
${ }^{1}$ Clinical Department of Ruminant, \\ University of Liège, Liège, Belgium \\ ${ }^{2}$ Gestion et Prévention de Santé, Regional \\ Association of Health and Animal \\ Identification, Ciney, Belgium \\ ${ }^{3}$ Department of Animal Production, \\ University of Liège, Liège, Belgium \\ Correspondence \\ Salem Djebala, Clinical Department of Rumi- \\ nant, University of Liege, Quartier Vallée \\ 2, Avenue de Cureghem 7A-7D, Liège 4000, \\ Belgium. \\ Email:sdjebala@uliege.be
}

\begin{abstract}
Background: Parietal fibrinous peritonitis (PFP) is a complication of laparotomy in cattle, consisting of fluid and fibrin accumulation within a fibrous capsule between the parietal peritoneum and the abdominal muscles. Since scientific information on PFP is scarce, we aim to collect available information to help practitioners in its diagnosis and treatment, and to formulate research perspectives.

Methods: PubMed and GoogleScholar databases were scanned using "cattle" or "bovine", and one of the following keywords: "seroma", "parietal fibrinous peritonitis", "retroperitoneal abscess", or "wound infection".

Results: Although scientific information is often anecdotal, two recent larger studies shed more light on PFP symptoms, diagnosis and treatment. Symptoms vary according to the cavity's localisation and size, and include anorexia, weight loss and an inflammatory status. Rectal palpation is strongly indicative, but the definitive diagnosis is made by ultrasound. Trueperella pyogenes and Escherichia coli are frequently isolated germs, although it remains unclear whether they are primary or secondary agents. Good survival rates were reported after surgical drainage.

Conclusion: Although the diagnosis and treatment seem clear, the exact pathogenesis of PFP should be the focus of ongoing research. This can be achieved by epidemiological data analysis focusing on risk factors like surgery technique, housing and ration.
\end{abstract}

\section{1 | INTRODUCTION}

Laparotomies are carried out in cattle throughout the world. ${ }^{1}$ In Belgium, laparotomy is very common: over 500,000 elective caesarean sections (CS) are carried out per year due to fetomaternal disproportions in the Belgian Blue cattle breed (BBCB) ${ }^{2-4}$; Belgian rural veterinarians typically perform between 500 and 1000 CS per year. ${ }^{5,6}$ In dairy cows, gastrointestinal disorders such as a displaced abomasum are a common indication for laparotomy. ${ }^{7}$ Like any surgery, laparotomy holds a risk of pre-, peri- and postoperative complications. ${ }^{5,7-9}$ In Belgium, complications after CS even represent up to $70 \%$ of all liability issues in rural veterinary practice. $^{8}$

Among the numerous postoperative complications, infectious disorders in the peritoneum are perceived as being the most problematic. Generalised peritonitis is well recognised by practitioners and is thoroughly described in literature. ${ }^{9,10}$ Parietal fibrinous peritonitis (PFP) is a particular localised form of peritonitis, described as an accumulation of fluid and fibrin between the parietal peritoneum and the abdominal wall, extending into the flank or the abdominal or pelvic cavity. $4,7,9,11-14$ Veterinarians are regularly confronted with PFP cases, especially in Belgium, and PFP has been estimated to cause considerable damage to the sector. ${ }^{4}$ Unfortunately, the nomenclature on PFP is confusing and scientific information is very scarce, ${ }^{2,4,11,13,15,16}$ leaving little evidence for practitioners concerning the diagnosis, treatment, prognosis and prevention of PFP. In this article, we aim to review the scientific literature on all aspects of PFP, with a specific focus on useful elements from a practical point of view. Furthermore, we aim to formulate perspectives for further research on this subject.

\section{2 | METHODOLOGY}

We scanned the PubMed and GoogleScholar databases for studies in English, French or Dutch, using "cattle" or "bovine," and one of the following keywords: "seroma," "parietal fibrinous peritonitis," "retroperitoneal abscess," or "wound infection" (or their translations in French and Dutch). Papers were often in French or Dutch and were sometimes unavailable via the Liège University Library; in these cases, we 
contacted the authors for access to the full papers. The selected articles were carefully read to verify whether the manuscript dealt with PFP. Some articles mentioning a "seroma" actually described a serous fluid accumulation under the skin after surgery and had to be discarded, whereas others described a PFP but did so under the (inaccurate) term "seroma."

\section{3 | DEFINITION AND NOMENCLATURE OF THE PATHOLOGY}

The first report of a large localised fluid accumulation in a thick capsule between the parietal peritoneum and the muscles was published in $1973,{ }^{17}$ leading to the asssumption that it was a particular and localised form of peritonitis. Since then, this pathology has been described by authors and veterinarians using different terms, including "seroma," "(retro) peritoneal abscess" or "clapier péritonéal” (French). ${ }^{7,11-13,15}$ To avoid confusion, the term "parietal fibrinous peritonitis" has been proposed as a more accurate definition, considering the nature, content and localisation of the pathology, and has been used in recent scientific literature. . $^{2,9,14,18}$ The content of a PFP is indeed fibrinous (accumulation of serum and fibrin, often contaminated), which discards the use of the term "abscess" or "seroma." Peritonitis is defined as a localised or generalized inflammation of the peritoneum, resulting in a serous and fibrinous exudate. ${ }^{19}$ For the description of this pathology, an accumulation of fluid and fibrin within a capsule formed by a modified parietal peritoneum justifies the term "parietal peritonitis."

Although the peritoneum is clearly involved, the exact pathogenesis of PFP is unknown: it is not clear whether PFP is primarily a peritonitis, or rather a manifestation of deep surgical site infection, affecting the peritoneum. $^{7}$

\section{4 | PFP'S CAPSULE AND FLUID COMPOSITION}

The volume of the PFP cavity is highly variable and depends mainly on its localization and the time between the causal laparotomy and the diagnosis. Volumes of 5-50 $\mathrm{l}^{2,4,7,15,18}$ and a mean weight of $28.75 \pm$ $5.15 \mathrm{~kg}^{9}$ have been reported.

Macroscopically, the PFP capsule is composed of a 2 - to 3 -cm-thick fibrous layer of parietal peritoneum. The cavity contains a serous yellow, haemorrhagic or serohaemorragic exudate and sometimes gas, and a varying amount of fibrin (up to $6 \mathrm{~kg}$ ), usually attached to the capsule in a lamellar way. The contents can have a normal or foul smell. . $^{2,411,14,15,18}$ The peritoneal fluid of PFP is characterised by a protein concentration above $30 \mathrm{~g} / \mathrm{L}$, which classifies it as an inflammatory exudate. A high lactate concentration and a low glucose concentration are often observed, indicative of bacterial proliferation. ${ }^{9}$ Cytologically, the PFP fluid has a very high proportion of neutrophils and, to a lesser extent, macrophages and lymphocytes. ${ }^{7}$ The simultaneous presence of fibrin, exudate and leucocytes in the PFP and the thickness of the connective tissue capsule indicate that PFP is a chronic inflammatory process, ${ }^{2,14,15,18}$ as also suggested by the blood profile (see below). Recent studies have clearly shown that the PFP fluid is often contaminated, ${ }^{7,9,13,14,16}$ in contrast to previous assumptions that PFP is sterile..$^{2,4,18}$

\section{5 | EPIDEMIOLOGY}

It is generally assumed that PFP is always the consequence of a laparotomy, ${ }^{2,4,11,13,15,18}$ although it has been reported in one BBCB heifer without a history of surgery, presumably due to a trauma inflicted during insemination. ${ }^{14}$ Clinical signs occur around 4 weeks after the initial laparotomy, although this interval may vary from 1 up to 7 weeks after surgery, $2,7,9,11,15,18$ and partly depends on the alertness of the farmer and the veterinarian. It is often assumed that PFP is detected earlier in dairy than in beef cows, since milk drop is a common early clinical sign. ${ }^{12}$ However, recent studies showed no difference in the interval between surgery and clinical signs between beef and dairy breeds. ${ }^{7,9}$ A PFP can occur in both beef ${ }^{9,13,14}$ and dairy cattle $7,12,15,18$ and can affect animals of any age or parity. ${ }^{7,9}$ In Belgium, PFP is described more often than in other countries, due to the large number of elective CS. ${ }^{2,4,9}$ It has been estimated that PFP occurs in $0.7-1 \%$ of CS. ${ }^{2,4,11}$ Logically, PFP is more commonly described in females than males, since the main indications for laparotomy in cattle (dystocia and abomasal dislocation) occur uniquely or far more commonly in cows than in bulls.

Practitioners often have the impression that PFP occurs without a clear cause; studies considering specific risk factors for PFP are very scarce. In one study, referred cases of PFP generally had no history of surgical complications during CS, in contrast to cows with generalized peritonitis. ${ }^{9}$ Selenium deficiency has been suggested as a risk factor for $\mathrm{PFP},{ }^{13}$ although this seems speculative due to the low number of cases in this study. Nevertheless, selenium deficiency is very common in beef cattle, ${ }^{20}$ and this hypothesis demands further exploration. Other potential risk factors, such as surgical technique, suture material, infectious status of the animal and the herd, nutrition or housing conditions, have not been studied.

\section{6 | AETIOLOGY AND PATHOGENESIS}

The exact pathogenesis of PFP is difficult to unravel since much time can pass between the onset and the diagnosis. ${ }^{2,7,12,18}$ It is generally accepted that PFP is an inflammatory reaction of the peritoneum to an event linked with surgery. ${ }^{2,11,12,13}$ The concrete trigger could be an infectious agent (viral, bacterial, fungal), a 
foreign body (suturing material), or an irritating stimulus (antibiotic, antiseptic or anaesthetic products; physical trauma or irritation). $4,7,9,13,14,18$

PFP has some similarities with sclerosing encapsulating peritonitis (SEP), a complication of peritoneal dialysis and abdominal surgery described in humans, ${ }^{21-23}$ dogs, ${ }^{24}$ and cats. ${ }^{25}$ The main risk factors for SEP are repeated laparoscopic interventions, unphysiological composition of dialysis fluids and disinfectants, perturbing the normal peritoneal histology, and resorption capacities. ${ }^{21,23,26}$ This finally leads to the accumulation of a modified transudate containing neutrophils, lymphocytes, and monocytes in a thickened parietal peritoneum ${ }^{24,27}$; the process can be contaminated. Some authors have suggested a genetic predispotition for SEP: certain individuals might have a different inflammatory cascade in response to irritating stimuli. ${ }^{26}$

In contrast to SEP, which is often a sterile inflammation of the peritoneum, ${ }^{22,26}$ PFP is contaminated in the majority of clinical cases. Various infectious agents have recently been isolated from PFP fluids, including Trueperella pyogenes, Escherichia coli, Clostridium perfringens, Bovine Herpesvirus 4 (BoHV4), Mycoplasma bovis, Coxiella burnetii, and Aspergillus fumigatus. ${ }^{7,9,13,16,28}$ The large diversity in these pathogens indicate that several ways of endogenous or exogenous contamination are possible., ${ }^{7,29}$ It is unclear whether these agents are primary aetiological causes of the PFP, or secondary contaminants of an initially sterile fluid pouch caused by surgery. In a recent study, BBCB cows suffering from PFP and generalized peritonitis were compared; the latter is generally considered as the result of contamination during surgery. Peritoneal samples of PFP and generalized peritonitis cows contained the same pathogens, suggesting contaminated surgery as a primary cause of both disorders. ${ }^{9}$ In contrast, another study reports the isolation of the same $M$. bovis strain from a PFP cavity, an arthritis, and a mastitis in the same cow, suggesting a haematogenous contamination of the PFP cavity from other infectious sites in the body. ${ }^{13}$ This may also be the case for other infectious agents found in PFP that can spread via the bloodstream, such as $C$. burnetii and BoHV4. Altogether, recent studies clearly demonstrate that PFP is not a sterile process, but the exact role of the aetiological agents remains unclear.

\section{7 | THE LOCALISATION AND CLINICAL SIGNS OF PFP}

Although the PFP always originates from the space between the parietal peritoneum and the muscles at the surgical site, ${ }^{2,4,7,15}$ multiple localisations have been described. The PFP cavity can be localised in the flank near the surgical wound, ${ }^{12,15}$ can form a cavity lined by parietal peritoneum extending into the abdominal or pelvic cavity or both, or can even cross the retroperitoneal space to the opposite flank of the initial surgery. ${ }^{9}$ Bourdette and coworkers $(2017)^{18}$ have described one case where a PFP cavity seemed to be localised between the visceral organs and isolated from the parietal peritoneum.

Clinical signs of PFP gradually appear after the initial surgery and worsen with time, ${ }^{4,11,13}$ and depend on the size and the localisation of the process and the degree of inflammation or infection. Slight pyrexia and increased respiratory and heart rates have been described in some but not all cows with PFP. $2,4,7,12,14$ Severe dehydration (more than 10\%) was reported in all cows enrolled in one study, ${ }^{9}$ while two-thirds of the patients described in another study ${ }^{7}$ had a normal hydration status. It should be noted that both clinical studies mentioned above describe patients being referred to a university hospital; therefore, previous treatments by a referring vet or transport may have modified clinical findings at the time of arrival.

The most common clinical signs of cows with PFP are anorexia and weight loss, gastrointestinal hypomotility, and maldigestion or diarrhoea. Distention of the paralumbar fossa, an arched back, and signs of colic may be present. These clinical signs can be explained by the presence of a large mass in the abdomen or the pelvis, an inflammatory condition, or adhesions between organs. . $^{4,9,12,14,15}$

\section{8 | DIAGNOSIS}

The typical history of a laparotomy in the weeks before the consultation, together with the clinical signs, raises the suspicion of PFP. 2,9,12,14,15 Certain additional elements of the clinical examination may increase the likelihood of PFP, for example when rectal palpation reveals reduced arm mobility and the presence of a firm or depressible fluctuating mass in the abdomen, flank, or pelvis. ${ }^{2,12,14,15,18}$ Auscultation and percussion may reveal a ping if a fluid and gas-filled PFP cavity is localised in the flank. ${ }^{4,30}$ Blood analysis of PFP patients are usually indicative of a chronic inflammatory status: haematology often reveals slight anaemia $^{7}$ and neutrophilia, while biochemical blood analysis often reveals hyperglobulinaemia, high fibrinogen concentrations, and delayed glutaraldehyde coagulation times. ${ }^{9,12,14,15,31}$

Although suggestive, anamnesis and clinical findings do not allow a conclusive diagnosis. General signs of PFP may resemble those of traumatic reticuloperitonitis ${ }^{15,32}$ or generalized peritonitis. ${ }^{9,32,33}$ Depending on its localisation, a PFP cavity in the flank, abdomen, or pelvis can easily be confused with peritoneal or abdominal abscesses, tumours, haematomas, and omental bursitis. ${ }^{18,34}$ Also, interor intramuscular abscesses and seromas and diffuse surgical site infection should be ruled out. ${ }^{15,32,35}$ A distended left or right paralumbar fossa with the eventual presence of a ping may be confused with a caecal or abomasal dilatation. ${ }^{32}$

Transabdominal or transrectal ultrasound with a 3.5 or $5 \mathrm{MHz}$ probe confirms the diagnosis in case of a PFP suspicion. The key diagnostic element of PFP is the presence of an anechogenic fluid and echogenic fibrin strands inside a thick fibrous capsule formed 
by the parietal peritoneum..$^{9,14,33,36}$ Ideally, both approaches are combined to obtain a good localization and visualization of the mass and an evaluation of displaced organs. ${ }^{4,9,13-15,18,31}$ The diagnosis of PFP is straightforward in cases where a large fluid filled cavity is easily found ${ }^{11-13,15}$ but can be challenging in case of a small volume or multiple or atypical localizations. ${ }^{18}$

Although unnecessary to confirm the diagnosis, collection of PFP fluids by paracentesis may add valuable elements to the treatment choice and prognosis. ${ }^{9,14,16,37}$ An aseptic (ultrasound guided) paracentesis enables a macroscopic, biochemical, and bacteriological analysis of the PFP fluid. ${ }^{15,16,18,36}$ An explorative laparotomy is the ultimate confirmation of the diagnosis and allows simultaneous drainage of the cavity. $7,9,11,14,15$

\section{9 | TREATMENT AND PROGNOSIS}

It is crucial to make a correct and complete diagnosis before starting the therapy. Euthanasia should be considered before or during treatment in patients with a low body condition score, a poor general condition, the presence of other pathologies, or in case of therapy failure. ${ }^{7,13,18}$

The principal element of the therapy is to eliminate any accumulated fibrin and tissue debris and to leave the wound open in order to drain the cavity. Generally, a surgical intervention is performed, on a standing patient, under local anaesthesia. The abdominal wall, muscular layers, and finally the PFP capsule are incised at the lowest ventral point defined by ultrasound examination, large enough to facilitate

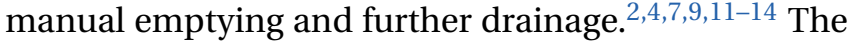
cavity is flushed profusely with a diluted antiseptic solution, for example chlorhexidine $0.05 \%$. $^{7,9,12-14}$ Flushing frequency is high at the onset of therapy (twice daily) and is reduced (once daily) according to the shrinking of the cavity and wound healing by secondary intention. . $^{2,11,13}$ The wound treatment is very long, varying from 20 days $^{12}$ to 5 weeks. ${ }^{4,13}$ Surgery and aftercare can be successfully carried out on farm to reduce treatment costs. ${ }^{7,12}$

Systemic antibiotic use is usually performed ${ }^{4,9,12-14}$ and is, ideally, based on a bacteriological culture and antibiotic susceptibility. ${ }^{14,37}$ One should bear in mind that antibiotic therapy may fail due to the impermeability of the fibrous capsule and the reduced perfusion of the target tissue. ${ }^{12,13}$

Some authors describe the use of non-steroid antiinflammatory drugs, mainly to improve appetite and the general condition. ${ }^{9,12,14}$ Intravenous fluid therapy (Nacl $0.9 \%$ ) during surgical treatment of PFP has been described to correct a fluid and acid/base imbalance $^{9,14}$ and to avoid hypovolemic shock while draining a large cavity. ${ }^{7}$

Clinical outcomes of PFP treatments reported in literature are variable. In a recent study, 30 of 32 dairy cows referred for PFP were successfully treated and discharged from the clinic. ${ }^{7}$ In BBCB cows, the mortality rate of PFP after treatment has been estimated to be around $13 \% .^{2,4}$ Recently, a long-term survival rate of eight of 12 referred cases of PFP in BBCB cows has been reported. ${ }^{9}$ It should be noted that this report described referred cases only, which may be seen in a more advanced stage than the cases first encountered in the field. A short interval between the initial surgery and the detection and treatment of the complication seems to improve the recovery and survival rate. ${ }^{11,12}$

Even if an animal completely recovers after treatment, culling should be considered because infertility and other problems may occur. ${ }^{7,12,14,15}$ In a recent study, among 12 BBCB cows treated for PFP, one was euthanized after the start of treatment, three died after being discharged from the clinic, three were successfully rebred, and five were successfully slaughtered with normal carcass weights after PFP recovery. ${ }^{9}$ Considering dairy cows, whenever data were available, patients discharged after PFP treatment seemed to reach their normal level of milk production in the current and future lactations. ${ }^{\text {? }}$

\section{0 | CONCLUSION AND PERSPECTIVES OF RESEARCH}

Until recently, reports on PFP in literature were scarce, often anecdotal and suffered from a lack of consistency in the nomenclature of the disease. Recently, two larger clinical studies on PFP in beef and dairy cows have been published, providing very useful information for practitioners concerning the clinical, diagnostic, therapeutic, and prognostic aspects on PFP. However, the pathogenesis of this disorder remains very unclear. An epidemiological data analysis on potential risk factors such as surgery technique, suture material, complications during surgery, housing conditions and ration, and their effect on the incidence of PFP would further help researchers and practitioners to correctly understand and prevent this complication.

\section{AC K N OWLED G E M E N T S}

We would like to thank everyone who contributed to the correction and the improvement of the article contents.

\section{CONFLICT OF INTEREST}

The authors declare no conflict of interest.

\section{O R C I D}

Salem Djebala (D) https:/ / orcid.org/0000-0003-33118219

Linde Gille (D) https: / / orcid.org/0000-0003-3720-9169

\section{RE F E R E N C E S}

1. Van Metre DC, Callan RJ, Holt TN, Garry FB. Abdominal Emergencies in Cattle. Vet Clin Food Anim. 2005;21:655-96.

2. Lamain G, Touati K, Rollin F. Aseptic fibrinous parietal peritonitis in cattle: retrospective and clinical study. In Proceedings of the 1st European Buiatrics Forum Conference, 1-3 December, Marseille, France. 2009.

3. Kolkman I, Aerts S, Vervaecke H, Vicca J, Vandelook J, De Kruif A, et al. Assessment of differences in some indicators of pain in 
double muscled Belgian Blue cows following naturally calving vs. caesarean section. Reprod Dom Anim. 2010;45:160-7.

4. Lamain G., Touati K., Rollin F, La peritonite fibrineuse partiale: Une complication dans l'espèce bovine. In Société nationale des groupements techniques vétérinaires, Proceedings of the Groupement Technique Vétérinaire Conference, 23-25 May, Nante, France. 2012.

5. Kolkman I, Opsomer G, Lips D, Lindenbergh B, De Kruif A, De Vliegher S. Pre-operative and operative difficulties during bovine caesarean section in Belgium and associated risk factors. Roprod Dom Anim. 2010;45:1020-7.

6. Djebala S, Moula N, Bayrou C, Sartelet A, Bossaert P. Prophylactic antibiotic usage by Belgian veterinarians during elective caesarean section in Belgian blue cattle. Prev Vet Med. 2019;172: 104785 .

7. Ferraro S, Desrochers A, Nichols S, Francoz D, Babkine M, Lardé $\mathrm{H}$, et al. Clinical characteristics, treatment, and outcome for cattle that developed retroperitoneal abscesses following paralumbar fossa laparotomy: 32 cases (1995-2017). JAVMA. 2019;256:814-21

8. Hanzen $\mathrm{C}$, Lourtie $\mathrm{O}$, Ectors $\mathrm{F}$. La césarienne dans l'espèce bovine. Ann Méd Vet. 1999;143:65-90.

9. Djebala S, Evrard J, Moula N, Gille L, Bayrou C, Eppe J, et al. Comparison between generalised peritonitis and parietal fibrinous peritonitis in cows after caesarean section. Vet Rec. 2020;187:e49.

10. Fecteau G. Management of peritonitis in cattle. Vet Clin Food Anim. 2005;21:155-71

11. Mijten P. Puerperal complications after cesarean section in dairy cows and in double- muscled cows. Reprod Dom Anim. 1998;33:175-9.

12. Nickol JM, Billerey M. Drainage d'un abcès péritonéal en pratique Chirurgie bovine. Point Vét. 2008;282:73-7.

13. Gille L, Pilo P, Valgaeren BR, Van Driessche L, Van Loo H, Bodmer M, et al. A new predilection site of Mycoplasma bovis: postsurgical seromas in beef cattle. Vet Microbiol. 2016;186: 67-70

14. Djebala S, Evrard J, Moula N, Sartelet A, Bossaert P. Atypical case of parietal fibrinous peritonitis in a Belgian Blue heifer without a history of laparotomy. Vet Rec Case Rep. 2020;8:e001086.

15. Braun U, Pusterla N, Anliker H. Ultrasonographic findings in three cows with peritonitis in the left flank region. Vet Rec. 1998;142:338-40.

16. Evrard J, Houtain GY, Gregoire F, et al. Pathogens associated with postsurgical seromas in cattle. In Proccedings of 5th European Buiatrics Forum Poster, 4-6 October, Bilbao, Spain. 2017.

17. Desbrosse F, Desbrosse H, Desbrosse M. Complication de la péritonite localisée chez la vache à la suite d'opération césarienne par le flanc gauche. Bull Mens Soc Vet Prat Fr. 1973;57:167-9.

18. Bourdette P, Mirbach S, Druart S, Hoolbecq M, Plassard V, ElBay S, et al. Clapier collé": un cas original de péritonite chez une montbéliarde en France. Point vét. 2017;372:44-7.

19. Sadeghian S. Kojouri G, Eftekhari Z, et al. Study of blood levels of electrolytes of infected cattle with peritonitis in comparison with healthy cattle. Intern J Appl Res Vet Med. 2011;9: 204-10.

20. Guyot H, Rollin F. Le diagnostic des carences en sélénium et iode chez les bovins. Ann Méd Vét. 2007;151:166-91.
21. Cnossen TT, Konings CJAM, Kooman JP, Lindholm B. Peritoneal sclerosis aetiology, diagnosis, treatment and prevention. Nephrol Dial Transplant. 2006;21:ii38-41

22. De Freitas D, Augustine T, Hurst H, Taylor P, Williams R, Hutchison A, et al. Encapsulating peritoneal sclerosis following renal transplantation despite tamoxifen and immunosuppressive therapy. Nephrol Dial Transplant. 2008;5:333-5.

23. Shahbazov R, Talanian M, Alejo JL, Azari F, Agarwal A, Brayman KL. Surgical management of encapsulating peritoneal sclerosis: a case report in kidney transplant patient. Case Rep Surg. 2018;8-11.

24. Etchepareborde S, Heimann M, Cohen-Solal A, Hamaide A. Use of tamoxifen in a German shepherd dog with sclerosing encapsulating peritonitis . J Small Anim Pract. 2010;51:649-53.

25. Sonck L, Chiers K, Ducatelle R, Van Brantegem L. Encapsulating peritoneal sclerosis in a young cat. Vet Rec Case Rep. 2018;6:14.

26. Jagirdar RM, Bozikas, A, Zarogiannis SG, Bartosova M, Schmitt CP, Liakopoulos V. Encapsulating peritonitis sclerosis: pathophysiology and current treatment options. J Mol Sci. 2019;20:5765.

27. Viega-Paraga $T$, Hecht $S$, Craig L. Imaging diagnosis-sclerosing encapsulating peritonitis in a dog. Vet Radiol Ultrasound. 2015;56:E65-9.

28. Djebala S, Evrard J, Gregoire F, Thiry D, Bayrou C, Moula N, et al. Infectious agents identified by real-time PCR, serology and bacteriology in blood and peritoneal exudate samples of cows affected by parietal fibrinous peritonitis after caesarean section. Vet. Sci. 2020;7:134-44.

29. Mijten P, Van den Bogaard AE, Hazen MG, et al. Bacterial contamination of fotal fluids at the time of caesarean section in the cow. Theriogenology 1996;97:513-21.

30. Vörös K, Bakos Z, Lukács Z, Tóth J, Szeredi L, Vetési F. Paraintestinal mesenteric abscess and chronic peritonitis in a bull. J Am Vet Med Assoc. 1997;211:1571-2.

31. Mohamed T, Oikawa S. Ultrasonographic characteristics of abdominal and thoracic abscesses in cattle and buffaloes. J Vet Med. 2007;517:512-7.

32. Cockcroft P, Jackson P. Clinical examination of the abdomen in adult cattle. In Practice 2004;26:304-17

33. Scott P. Abdominal ultrasonography as an adjunct to clinical examination 2 cattle. In Practice J Vet Med. 2012;34:66-72.

34. Braun U. Ascites in cattle ultrasonographic findings and diagnosis. Vet Clin Food Anim. 2016;32:55-83.

35. Bienek A, Grunert E. Sonographic control of wound healing after caesarean section in cattle. Dtsch Tierarztl Wochenschr. 1997;104:423-7.

36. Braun U. Ultrasound as a decision-making tool in abdominal surgery in cows. Vet Clin Food Anim. 2005;21:33-53.

37. Tulleners E. Prevention and treatment of complications of bovine gastrointestinal surgery. Vet Clin North Am Food Alim Pract. 1990;6:495-514.

How to cite this article: Djebala S, Evrard J, Moula $\mathrm{N}$ et al. Parietal fibrinous peritonitis in cattle: A literature review Vet Rec Open. 2021;e30. https://doi.org/10.1002/vetr.30 